\title{
Measurements of Carbon ion fragmentation on a thin gold target by the FIRST collaboration at GSI
}

\author{
Francesca Balestra* \\ Istituto Nazionale di Fisica Nucleare - Sezione di Torino, Italy; Dipartimento di Fisica, \\ Politecnico di Torino, Italy \\ E-mail: francesca.balestra@polito.it
}

On behalf of the FIRST collaboration.

The FIRST (Fragmentation of Ions Relevant for Space and Therapy) experiment, carried out at GSI laboratory, is designed and built by an international collaboration from France, Germany, Italy and Spain. The experiment aims to study the interactions of a ${ }^{12} \mathrm{C}$ ion beam with thin targets, in particular to measure the double differential cross section related to the fragmentation of carbon ions. The first set of data was taken using, as projectiles, ${ }^{12} \mathrm{C}$ ions of energy 400 $\mathrm{MeV} /$ nucleon. The experimental setup consists of two parts: the forward region, dedicated to the measurement of the fragments emitted at low polar angle $\left(\lesssim 6^{\circ}\right)$ with respect to the beam direction and the interaction region, whose detectors aim to measure the light fragments covering a larger polar angle range (up to $40^{\circ}$ ). The reconstruction algorithms were tuned and validated using the FLUKA simulation software. The results, in terms of differential cross sections as a function of angle and energy, are reported here.

53rd International Winter Meeting on Nuclear Physics,

26-30 January 2015

Bormio, Italy

\footnotetext{
* Speaker.
} 


\section{Introduction}

The study of the fragmentation processes is relevant in different Physics fields concerning both basic research and applications. The FIRST (Fragmentation of Ions Relevant for Space and Therapy) experiment at SIS accelerator of GSI laboratory in Darmstadt, has been designed to measure the ion fragmentation cross sections in the energy range 100-1000 MeV/nucleon. The experiment is performed by an international collaboration made of institutions from Germany, France, Italy and Spain. The energy range explored by FIRST is of fundamental importance for space radiation shielding and hadrontherapy applications and is interesting for different aspects concerning nuclear physics. High-quality experimental data concerning ${ }^{12} \mathrm{C},{ }^{16} \mathrm{O}$ and ${ }^{56} \mathrm{Fe}$ fragmentation on different targets are still missing in literature. Furthermore, a deeper understanding of the reaction mechanisms is fundamental for the development of reliable nuclear interaction models to be used in simulation codes. The interaction and transport of high-energy heavy ions in matter is a subject of interest in the fields of astrophysics, radiobiology, radiation medicine, and radiation protection [1]. An accurate description of the fragmentation of heavy ions is important to understand the effects of the high $\mathrm{Z}$ component of galactic cosmic radiation (GCR) on humans in space [2], for radiationinduced damage into microelectronics circuits and for shielding in accelerator environments.

Moreover, the interaction and transport of light energetic ions ( $\mathrm{Z}<9$ and $\mathrm{E} \leq 400 \mathrm{MeV} /$ nucleon) in tissue- like matter is extremely important for cancer therapy with charged particles, a field in rapid expansion and pioneered in Europe at GSI [3]. Normally, less than 50\% of the carbon projectiles actually reach the tumor in therapy: hence, a precise knowledge of the fragmentation cross-sections is necessary for treatment planning [4]. Both in hadrontherapy and space radiation protection, computer codes are used to calculate the beam transport in matter [5]. The total and partial fragmentation cross-sections are the crucial parameters of the transport codes.

The FIRST collaboration principal aim is to measure the double differential fragmentation cross section (DDCS) of ${ }^{12} \mathrm{C}$ ions, on thin targets of gold and other materials, at $400 \mathrm{MeV} /$ nucleon [6], a energy for which, at present, experimental data are missing.

About 4 million events of collisions between ${ }^{12} \mathrm{C}$ and the gold target were collected during the data taking in 2011: technique and results are shown in the following chapters.

\section{The FIRST experiment}

The experimental setup of FIRST is shown in Fig. 1. It is divided into two regions: the Interaction Region (IR) [7] and the Large Detector Region separated by a magnet working as a spectrometer. The impinging beam and created fragments are detected in the IR within some centimeters from the target; beyond the magnet, in the Large Detector Region, the fragments are detected using devices with typical dimensions of meters. Following the beam path, it is possible to see the following detectors. The Start Counter (SC), made of a thin layer of plastic scintillator, was used as minimum bias trigger of the data acquisition. A pixel silicon detector, Vertex (VTX), placed just behind the target, allows a precise reconstruction of the tracks of the fragments produced in the target and of their production vertex. The long read-out time of this pixel detector and the beam rate in the $1-10 \mathrm{kHz}$ range required the development of a dedicated fast Beam Monitor (BM) detector. It consists of a drift chamber which, by providing the position of the impinging ${ }^{12} \mathrm{C}$ ion in the 


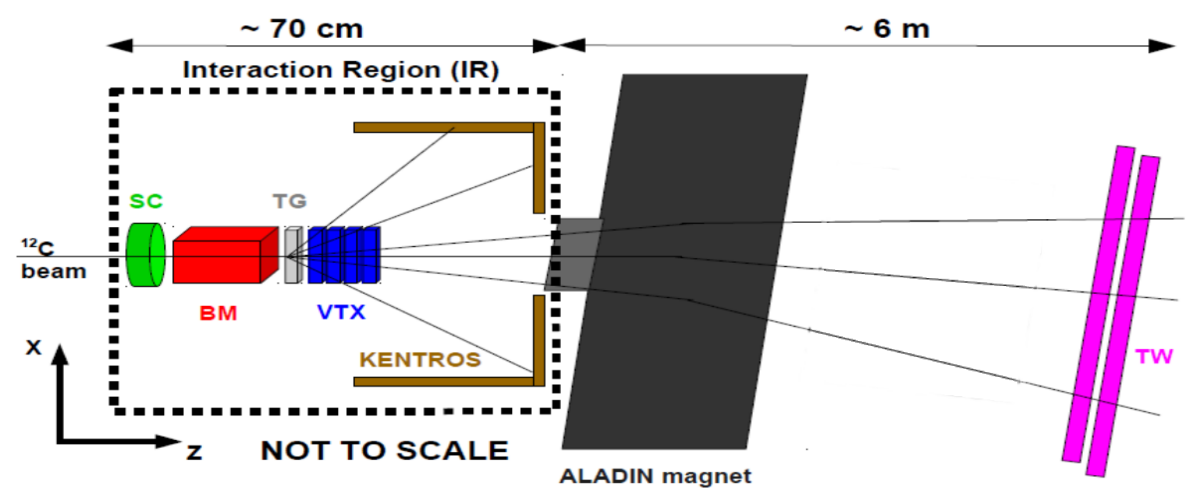

Figure 1: Sketch of the FIRST experiment layout.

target, is capable of resolving the event Pile Up (PU) ambiguity in the VTX. A plastic scintillator calorimeter (Kinetic ENergy and Time Resolution Optimized on Scintillator, KENTROS) has been used to detect fragments, mainly protons and helium ions, emitted at large angles. This detector, with cylindrical symmetry, surrounds the target and the vertex detector, covering a polar angle between $6^{\circ}$ and $90^{\circ}$.

About all the heavy fragments $(\mathrm{Z}>2)$ and a significant fraction of lighter ions $(\mathrm{Z}=1$ and $\mathrm{Z}=2)$ are produced in the forward direction $\left(\theta \leq 6^{\circ}\right)$ and travel within the magnetic acceptance of the magnet, a dipole called ALADiN, with vertical field. The charged fragment momentum is computed by tracking the fragment trajectory in the horizontal plane. Beyond the magnetic region, fragments enter in the Large Detector Region where, after a flight of about 6 meters, are detected by the Time of Flight Wall (ToF-Wall - TW). This is a large area system of scintillators that provides the measurement of the impinging point, the arrival time and the energy release of the particles. This information allows a clean separation of fragments with different charge.

The results described here only concern the polar angle region $<6^{\circ}$, corresponding to the forward direction, within the ALADiN magnet acceptance. A more detailed description of VTX and TW detectors is given in the following.

\subsection{The Vertex detector: VTX}

The purpose of the Vertex detector (VTX) is the trajectory and vertex reconstruction of fragments produced in the target. The Vertex detector has to fulfill several requirements [8]: a wide angular acceptance is required to track also large angle projectiles (outside the ALADiN acceptance window) and a good angular resolution on tracks (about $0.3^{\circ}$ ) is needed to achieve a good fragment separation in the forward direction. Moreover, the whole detector thickness should not exceed a few per cent of the target thickness to keep the probability of fragmentation inside the sensors at the few per cent level. In order to satisfy all the requirements, a new pixel sensor, called Mimosa26 (Minimum Ionizing Metal Oxide Semiconductor Active pixel sensor, M26), has been developed $[8,9]$ to equip VTX. VTX is made of 4 stations. On each of them, two M26 sensors are glued on the two sides of a $1 \mathrm{~mm}$ thick PCB (Printed Circuit Board) in correspondence to a square hole with sensing area of $2 \times 2 \mathrm{~cm}^{2}$ [6].

The tracking reconstruction efficiency of this detector, evaluated on Monte Carlo simulation 
events, is $98.7 \pm 0.1 \%$, with a measured proportion of fake tracks of $1.99 \pm 0.01 \%$. Furthermore, the resolution of the vertex reconstruction, also evaluated using Monte Carlo events, is better than $10 \mu \mathrm{m}$ in $\mathrm{X}$ and $\mathrm{Y}$ directions and better than $50 \mu \mathrm{m}$ in $\mathrm{Z}$ direction [10]. Figure 2 shows the residuals obtained using ${ }^{12} \mathrm{C}$ ion events at $400 \mathrm{MeV} /$ nucleon for $\mathrm{X}$ and $\mathrm{Y}$ coordinates. The residual distribution was used to evaluate the resolution $(\sigma)$ of the tracking device by means of a Gaussian fit. The resolution in $\mathrm{X}$ and $\mathrm{Y}$ directions is better than $\sigma=5 \mu \mathrm{m}$.
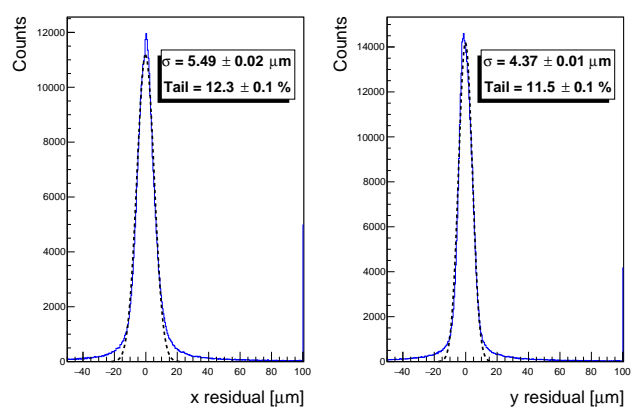

Figure 2: Residuals obtained for reconstructed tracks in the $\mathrm{X}$ (left plot) and $\mathrm{Y}$ (right plot) directions. The data distribution is shown in blue, the result of a Gaussian fit to the histogram is superimposed in black.

The VTX detector is able to provide also information on the fragment charge: it exploits the correlation between the size of the hit clusters in the VTX detector and the fragment charge. To benchmark the capabilities of the VTX detector, a clean sample of fragments whose charge was obtained from the TW detector has been used to estimate the correlation with the cluster size. The result is shown in Fig. 3.

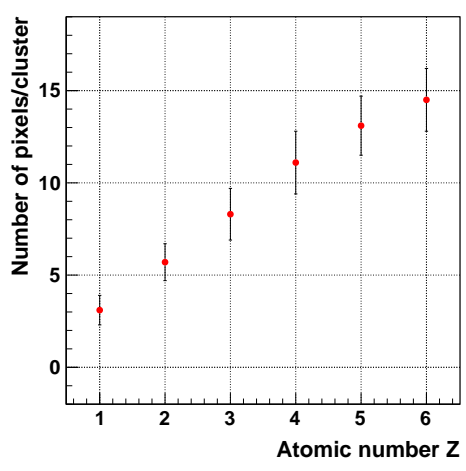

Figure 3: Number of pixel associated to a given VTX cluster, as a function of the fragment charge as assigned from the TW detector reconstruction algorithm.

\subsection{The ToF-Wall detector: TW}

The ToF-Wall is basically the spectrometer of FIRST, for the forwardly emitted particles. It measures the arrival time, energy release and impinging position of the fragments produced with polar angles smaller than $\sim 6^{\circ}$, allowing the reconstruction of their impulse and charge. It is placed at a distance of about 6 meters from the target. The TW consists of two layers, Front and Rear, 
made of 12 modules, each containing 8 BC-408 plastic scintillator slats $\left(110 \times 2.5 \times 1 \mathrm{~cm}^{3}\right)$. Each slat is connected to a photomultiplier tube (PMT) on each side [6]. Through the signals from the PMTs, read by ADCs and TDCs, the vertical coordinate, $\mathrm{Y}$, of the arrival position of the fragment on the TW, the arrival Time of Flight, ToF, and the energy lost, $\mathrm{E}_{\text {loss }}$, by the fragment in the TW, are evaluated. The resolution of these three fundamental quantities $\left(\mathrm{E}_{\text {loss }}, \mathrm{Y}, \mathrm{ToF}\right)$ is estimated by comparing the values of the corresponding hits in the Front and Rear planes. The Y and ToF resolutions are reported in Fig. 4, as an example, as a function of the released energy, for data and MC: the Y coordinate shows a strong dependence upon the released energy (varying from 2 to 5 $\mathrm{cm}$ ), while the ToF resolution is nearly constant (about $800 \mathrm{ps}$ ) except for values very close to 0 . It is remarkable the very good agreement with the simulated resolution in MC.

The horizontal coordinate, $X$, resolution derives from the slat width: $X_{r e s}=2.5 / \sqrt{12} \mathrm{~cm}=0.7 \mathrm{~cm}$. In the TW, the fragment charge identification is performed using the $\mathrm{E}_{\text {loss }}$ and ToF measurements
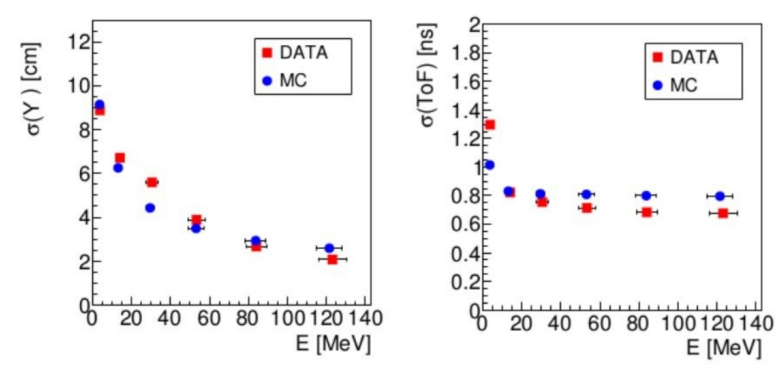

Figure 4: Left: Y resolution. Right: ToF resolution. The distributions are shown for data (red squares) and $\mathrm{MC}$ (blue circles) event samples as a function of the released energy.

of each fragment. In fact, in the $\mathrm{E}_{\text {loss }}-\mathrm{ToF}$ plane, it is possible to distinguish among six spots, related to different fragment charges (see Fig. 5). Each spot is fitted with the Bethe-Bloch formula

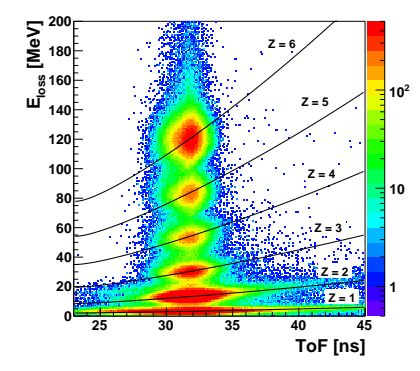

Figure 5: $\mathrm{E}_{\text {loss }} \mathrm{vsToF}$.

with $\mathrm{Z}$ corresponding to the spot charge. For each TW hit, the identification algorithm computes the normalized distance and assigns the charge corresponding to the one minimizing it to the fragment.

\subsection{The Monte Carlo code}

The Monte Carlo (MC) simulation developed for the FIRST experiment is built for several purposes, from the design and optimization of the experimental setup to the support for the reconstruction software development. In fact the simulation code is able to provide the simulated 
detector response data of an event. Moreover, it is fundamental in evaluating acceptances, reconstruction efficiencies and uncertainties.

For what concern the experimental setup, all the detectors and the ALADiN magnet have been implemented in the simulation considering their positions, the whole geometry and all the materials. This is necessary to properly evaluate the interactions in all the active detectors and the production of secondary particles in out-of-target fragmentation processes. The positions of the detectors in the experimental area are fixed on the basis of the results of optical survey measurements performed after the data taking, complemented with alignment studies.

The FIRST simulation exploits the multi-purpose FLUKA [11, 12, 13] MC code to simulate the particle transport and interactions with matter. In fact, FLUKA has been considered acceptable in the energy range of FIRST, to design the experiment, both for the description of electromagnetic processes and to provide a modeling of nuclear interactions (10\% of uncertainty) [13].

\subsection{The global reconstruction algorithm}

The tracking algorithm has the aim to determine the momentum and mass of all the charged particles produced in the ${ }^{12} \mathrm{C}$-target reactions. In the region considered in this study (polar angle $<$ $6^{\circ}$ ), the fragments are reconstructed using an iterative procedure that matches the VTX tracks and TW hits detected in each event.

The reconstruction is performed following three fundamental steps. At first, each event is selected applying filters based on the quality and quantity of the tracks inside VTX and of the TW hits. For each filtered event, a list of Global Track Candidates (GTC) is obtained, by combining all the VTX tracks with the TW hits: each VTX track is paired with one (or more) TW hit. A TW charge is assigned to each GTC and the track candidates are ranked in a list accordingly to a Scoring Function. For each GTC, the algorithm performs an iterative forward-tracking through the magnetic field: starting from the positions and angles measured in the IR, with a first-guess value for the momentum, the measured coordinates are forward-tracked inside the ALADiN magnetic field and up to the TW. The horizontal coordinate of the track projected on TW is compared with the one measured by the TW. If the two quantities differ one from the other more than $1.25 \mathrm{~cm}$, it means that the hypothesized momentum is not correct. Therefore it is changed and the forward-tracking procedure is repeated, until the reconstructed position of the hit agrees with the measured one. After the convergence, the algorithm returns, for each GTC, the reconstructed momentum. At this level, the Scoring Function (SF) is used to rank the GTC list and to select the best candidate for each event. The SF is based on weight factors coming from measured quantities: the fragment charge and vertical coordinate. Both the quantities are determined by the detectors TW and VTX.

Starting from the difference between the particle charge as reconstructed from the VTX and from the TW detectors $\left(\Delta_{c h g}\right)$ and the difference between the Y position $\left(\Delta_{Y}\right)$ as extrapolated from the VTX and as measured with the TW, the adopted SF is defined as:

$$
S F=\sqrt{\Delta_{c h g}^{2} * w_{c h g}^{2}+\Delta_{Y}^{2} * w_{Y}^{2}}
$$

$w_{c h g}$ and $w_{Y}$ are weight factors, optimized on the MC simulations, by minimizing the fraction of wrongly reconstructed tracks.

The tracking algorithm has an efficiency that has been evaluated using the full MC simulation 
sample available (50 million of events). The tracking efficiency is defined, in each energy and polar angle interval, as: $\varepsilon_{t r k}=\frac{n_{R e c}}{n_{\text {Prod }}}$ in which the properly reconstructed tracks $\left(n_{\text {Rec }}\right)$ are counted out of all the produced ones $\left(n_{\text {Prod }}\right)$.

\section{The cross section results}

Two differential fragmentation cross sections have been determined in the FIRST experiment: the energy and the solid angle differential cross sections

$$
\begin{aligned}
& \frac{d \sigma^{Z, A}}{d E}=\frac{Y_{i}^{Z, A}}{N_{{ }^{2} C} * N_{t, S} * \Delta E_{i} * \varepsilon_{t r k}\left(E_{i}\right)} \\
& \frac{d \sigma^{Z, A}}{d \Omega}=\frac{X_{j}^{Z, A}}{N_{{ }^{2} C} * N_{t, S} * \Delta \Omega_{j} * \varepsilon_{t r k}\left(\theta_{j}\right)}
\end{aligned}
$$

At the denominator of the ratio there are several elements: $N_{{ }^{2} C}$ is the number of carbon ions impinging on the target: $4.16^{*} 10^{6}$ events; $N_{t, S}$ is the surface nuclear density of the target: $\rho^{*} \mathrm{t}=0.96$ $\mathrm{g} / \mathrm{cm}^{2}$ ( $\rho$ is the target density, $\mathrm{t}$ is the target thickness); $\varepsilon_{t r k}(E)$ and $\varepsilon_{t r k}(\theta)$ are the tracking reconstruction efficiencies: $\varepsilon_{t r k}=\frac{n_{R e c}}{n_{\text {Prod }}} ; \Delta E_{i}$ is the normalized kinetic energy $\left(E_{\text {kin }} /\right.$ nucleon $)$ interval and finally $\Delta \Omega_{j}=2 \pi \Delta\left(\cos \theta_{j}\right)$ is the solid angle interval.

At the numerator of both the equations 3.1 and 3.2, the are the so called yields: $Y_{i}^{Z, A}$ and $X_{j}^{Z, A}$ are the number of fragments with charge $Z$ and mass $A$, detected in the energy and angular intervals $\Delta E_{i}$ and $\Delta \Omega_{j}$, respectively. The yields are calculated from the mass spectra through an extended unbinned maximum likelihood fit, using the RooFit ROOT toolkit [14]. In Fig. 6, the fit performed on the mass spectrum for the charge $\mathrm{Z}=2$ in the energy range between 200 and $230 \mathrm{MeV}$ /nucleon is shown, as an example: the total Probability Density Function (PDF), in red, is superimposed to the data (black dots). The combinatorial background, which takes into account the wrongly associated events in VTX and TW and the events badly reconstructed by the reconstruction algorithm, is reported in Fig. 6 as a dotted line. The background PDF shape is evaluated using the MC simulation and it is then normalized on the data values. The blue curve, finally, represents the PDF of the signal, modeled through Gaussian functions. In addition, the contribution of the so called

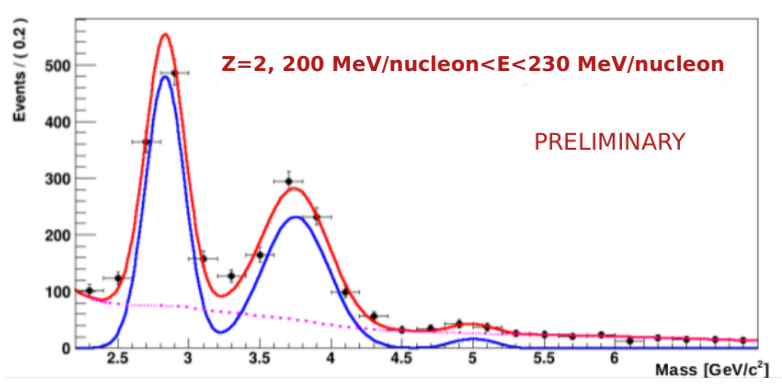

Figure 6: Fit result for the He fragment mass spectrum in the energy range 200-230 MeV/nucleon.

"cross-feed background" originating from the limited precision of the algorithm of the charge identification performed by the TW (described in section 2.2), has been taken into account using the MC simulations. 


\subsection{The kinetic energy and angular distributions}

The differential cross sections of the C-Au interaction are reported in the Figs. 7 and 8 as a function of the normalized kinetic energy and of the polar angle, respectively. For each charge Z, all the non negligible contributions from different isotopes are summed up. The uncertainties on

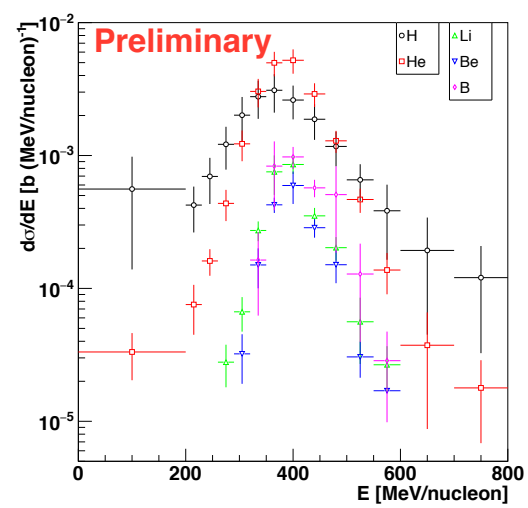

Figure 7: Energy Single Differential Cross Sections $\frac{d \sigma^{Z}}{d E}$ as a function of the normalized kinetic energy, for the $\mathrm{H}, \mathrm{He}, \mathrm{Li}, \mathrm{Be}, \mathrm{B}$ ions. The error bars take into account both the systematic and the statistic uncertainties. This distribution has not yet been unfolded to take into account the detector resolution.

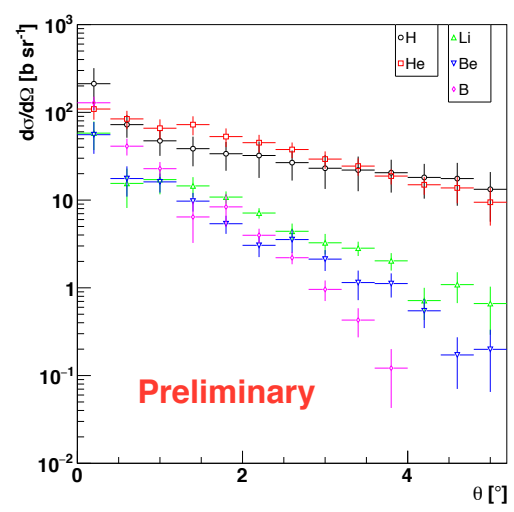

Figure 8: Angular Single Differential Cross Sections $\frac{d \sigma^{Z}}{d \Omega}$ as a function of the polar angle, for the $\mathrm{H}, \mathrm{He}, \mathrm{Li}$, $\mathrm{Be}, \mathrm{B}$ ions. The error bars take into account both the systematic and the statistic uncertainties.

the measured cross section values, shown in the plots 7 and 8 , include both the statistical and the systematic contributions. The systematic uncertainty is the main one. The largest contributions to the systematic uncertainty can be divided in three main categories:

a) uncertainties due to the PDF modeling in the yield evaluation; b) uncertainties due to the tracking algorithm and c) uncertainties on the detector positions and alignments.

Looking at the energy differential cross sections in Fig. 7, it can be observed that the distributions of all the charges are bell-shaped in semi-logarithmic scale, their widths, at the contrary, are wider for the lighter fragments $(\mathrm{H}, \mathrm{He})$. Since the highest charges $(\mathrm{Z}=3,4,5)$ are narrower distributed, it is possible to deduce that such fragments are mostly residuals of the ${ }^{12} \mathrm{C}$ projectiles, 
which almost conserve the normalized energy (and impulse). Another striking feature is the position of the peak of the distributions: for all the charges it is close to about $400 \mathrm{MeV} /$ nucleon, corresponding to the primary beam energy. In fact, the energy loss by the beam into the thin $\mathrm{Au}$ target has been estimated to be only $8 \mathrm{MeV}$, in average.

Other interesting features of the forward fragmentation can be observed looking carefully at the angular differential cross sections in Fig. 8: it is evident that the heaviest fragments $(Z=3,4,5)$ are more forwardly focused (within $2^{\circ}$ ) than $\mathrm{H}$ and He. Hence, considering the energy loss the heavier fragments release energy closer to the trajectory of the primary beam, with respect to $\mathrm{H}$ and $\mathrm{He}$.

Moreover, from both the Fig. 7 and 8, it can be seen that the lighter fragments ( $\mathrm{H}$ and $\mathrm{He}$ ) are the most abundant in the forward direction.

\section{Conclusions}

The FIRST experiment performed a measurement of SDCS, as a function of fragment angles and kinetic energies, studying the collisions of a ${ }^{12} \mathrm{C}$ beam impinging on a thin $(0.5 \mathrm{~mm})$ gold target. These measurements fill a gap in the previous data concerning the differential cross section of fragmentation of carbon ions at $400 \mathrm{MeV} /$ nucleon, particularly interesting for particle therapy and space applications.

The plots of the single differential cross sections pointed out some important preliminary outcomes. For what concerns the cross section as a function of the polar angle, the production of fragments falls down exponentially with increasing angle, except for the lighter isotopes $(Z=1,2)$, which are spread out over the whole angular acceptance $\left(0 \leq \theta \leq 6^{\circ}\right)$. This means that the lightest fragments are the main source of dose scattering far from the original beam direction; the heavier ions, instead, more forward emitted, contribute to the dose deposition close to the primary beam trajectory. The cross sections as a function of the kinetic energy, instead, showed a peak centered close to the beam energy (400 MeV/nucleon). This suggests that the greatest part of the detected particles comes from the projectile fragmentation.

\section{References}

[1] C. X. Chen et al., Interactions in hydrogen of relativistic neon to nickel projectiles: Total charge-changing cross sections, Phys. Rev. C, 49, (1994), 3200.

[2] M. Durante, Biological effects of cosmic radiation in low-Earth orbit, Int. J. Mod. Phys. A, 17, (2002), 1713.

[3] U. Amaldi, G. Kraft, Radiotherapy with beams of carbon ions, Rep. Prog. Phys., 68, (2005), 1861.

[4] D. Schardt, Tumor therapy with high-energy carbon ion beams, Nucl. Phys. A, 787, (2007), 683c.

[5] C. La Tessa et al., Test of weak and strong factorization in nucleus-nucleus collisions at several hundred MeV/nucleon, Nucl. Phys. A, 791, (2007), 434.

[6] R. Pleskac et al., The FIRST Experiment at GSI, Nucl. Instr. Meth. in Phys. Res. A, 687, (2012), 130.

[7] Z. Abou-Haidar et al., Performance of upstream interaction region detectors for the FIRST experiment at GSI, Journ. of Instr., 7, P02006, (2012). 
[8] E. Spiriti, M. De Napoli, F. Romano et al., The FIRST experiment: interaction region and MAPS vertex detector, Nuclear Physics B - Proceedings Supplements, 215, Issue 1, (2011), 157-161.

[9] http://www.iphc.cnrs.fr/-CMOS-ILC-.html

[10] R. Rescigno et al., Performance of the reconstruction algorithms of the FIRST experiment Pixel Sensors Vertex detector, Nuclear Inst. and Methods in Physics Research A, 767, (2014), 34-40.

[11] G. Battistoni et al., The FLUKA code: description and benchmarking, Proceedings of the Hadronic Shower Simulation Workshop 2006, AIP Conference Proceedings, 896, (2007), 31.

[12] A. Ferrari, P.R. Sala, A. Fassó, J. Ranft, FLUKA: A Multi Particle Transport Code, Technical Report CERN-2005-10, INFN/TC05/11, SLAC-R-773, (2005).

[13] T.T. Böhlen et al., Benchmarking nuclear models of FLUKA and GEANT4 for carbon ion therapy, Physics in Medicine and Biology, 55 (19), (2010), 5833.

[14] W. Verkerke and D. P. Kirkby, The RooFit toolkit for data modeling, arXiv: physics/0306116. 